\title{
Efficacy of various side-to-side toothbrushes for noncontact biofilm removal
}

\author{
Julia C. Schmidt • Monika Astasov-Frauenhoffer • \\ Irmgard Hauser-Gerspach • Jan-Philipp Schmidt • \\ Tuomas Waltimo • Roland Weiger • Clemens Walter
}

Received: 20 March 2013 / Accepted: 3 July 2013 / Published online: 20 July 2013

(C) Springer-Verlag Berlin Heidelberg 2013

\begin{abstract}
Objectives The aim of this study was to evaluate the efficacy of four different powered toothbrushes with side-to-side action for noncontact biofilm removal in vitro.

Materials and methods A three-species biofilm was formed in vitro on protein-coated titanium disks using a flow chamber combined with a static biofilm growth model. Subsequently, the biofilm-coated substrates were exposed to four different side-to-side toothbrushes (A, B, C, and D) with various brushing times $(2,4$, and $6 \mathrm{~s})$ and brushing (bristleto-disk) distances ( 0,2 , and $4 \mathrm{~mm})$. The biofilm volumes were measured using volumetric analyses with confocal laser scanning microscope images and Imaris version 7.5.2 software.

Results The median percentages of biofilm reduction by the analyzed toothbrushes ranged from $9 \%$ to $80 \%$. The abilities of the tested toothbrushes to remove the in vitro biofilm differed significantly $(p<0.05)$. Two of the tested toothbrushes $(\mathrm{C}$ and $\mathrm{D})$ were capable of significant biofilm reduction by noncontact brushing.

Conclusions It was possible to reduce a three-species in vitro biofilm by noncontact brushing with two out of four side-toside toothbrushes.

Clinical relevance Toothbrushes $\mathrm{C}$ and $\mathrm{D}$ show in vitro a high efficacy in biofilm removal without bristle contact.
\end{abstract}

J. C. Schmidt $\cdot$ R. Weiger $\cdot$ C. Walter $(\bowtie)$

Department of Periodontology, Cariology and Endodontology,

School of Dental Medicine, University of Basel, Hebelstrasse 3,

4056 Basel, Switzerland

e-mail: clemens.walter@unibas.ch

M. Astasov-Frauenhoffer · I. Hauser-Gerspach · T. Waltimo Clinic of Preventive Dentistry and Oral Microbiology, School of Dental Medicine, University of Basel, Basel, Switzerland

J.-P. Schmidt

Institute of Insurance Science, Faculty of Mathematics and

Economics, University of Ulm, Ulm, Germany
Keywords Side-to-side toothbrush · Biofilm • Hydrodynamic effect · Oral hygiene $\cdot$ Preventive dentistry

\section{Introduction}

The treatment of biofilm-associated diseases, which includes comprehensive periodontal or peri-implant therapy, is a challenge in human infectiology [1-3]. A biofilm is a microbial structure that adheres to wet surfaces [4]. From a clinical perspective, this complex structure plays a critical role protecting the associated microorganisms from both the host immune system and antimicrobial agents. It is commonly understood that a pathogenic oral biofilm needs to be disturbed by mechanical means, including self-performed daily oral hygiene [5-7].

A recent systematic review reported that powered toothbrushes with side-to-side, multidimensional, and ultrasonic actions can reduce biofilm in vitro by noncontact brushing [8]. Interactions among hydrodynamic phenomena, passing air bubbles, and acoustic energy transfer appear to contribute to noncontact biofilm removal $[9,10]$. Based on the current evidence, the authors of the review suggested that future research should consider (1) aspects of in vitro biofilm formation and (2) the relevance of experimental brushing protocols [8].

The strength of an in vitro biofilm depends on several parameters, including its initial adhesion to a surface, the acquisition of a salivary pellicle that provides receptors for bacterial binding, and the microbial growth time [11, 12]. The co-aggregation of multiple bacterial species and the presence of environmental factors, such as hydrodynamic effects, influence the cohesive forces within a biofilm [13, 14]. In addition to the oscillation rate of the toothbrush head, the brushing time, the distance between the toothbrush bristles and the tooth surface, and the presence of a liquid environment may affect the efficacy of a toothbrush [15, 
16]. Future in vitro studies of noncontact biofilm removal may therefore benefit from the use of multispecies biofilms grown under dynamic conditions on a suitable substratum coated with a salivary pellicle. It is essential to consider the possible translation of experimental findings to the clinical setting when studying different brushing parameters.

The aim of the present study was to determine the efficacy of four different powered toothbrushes with side-to-side action for the noncontact removal of a multispecies biofilm in vitro.

\section{Materials and methods}

\section{Biofilm formation}

A previously described protocol for multispecies biofilm formation was utilized [17]. Briefly, Streptococcus sanguinis DSM 20068, Fusobacterium nucleatum ATCC 10953, and Porphyromonas gingivalis DSM 20709 were grown in liquid broth, and the resulting bacterial suspensions were used for biofilm formation.

Sterile disks of commercially pure titanium (Grade 2, ASTM F-67) with a sandblasted/acid etched (SLA) surface, $5 \mathrm{~mm}$ in diameter and $1 \mathrm{~mm}$ in thickness (Straumann AG, Basel, Switzerland), were used as substrates. Prior to each experiment, the disks were placed in a serum/saliva mixture $(1: 10)$ at room temperature for $15 \mathrm{~min}$ to allow protein pellicle formation. Fasting-stimulated saliva from healthy volunteers was prepared according to an established protocol [18]. Before use, the saliva was mixed with pooled serum (Blutspendezentrum, Basel, Switzerland). The proteincoated substrates were placed in an anaerobic flow chamber (details of the flow chamber system have been previously described) [18-22]. The bacterial suspension was circulated at $0.8 \mathrm{ml} \mathrm{min}{ }^{-1}$ under anaerobic conditions (MACS MG; Don Whitley Scientific Ltd.) in an atmosphere of $80 \% \mathrm{~N}_{2}$, $10 \% \mathrm{H}_{2}$, and $10 \% \mathrm{CO}_{2}$ at $37^{\circ} \mathrm{C}$ for $72 \mathrm{~h}$ and was renewed at 24-h intervals. The disks were removed from the anaerobic flow chamber. The wells of a 12-well plate were filled with a mixture of thioglycolate (bioMerieux SA, Geneva, Switzerland) enriched with $5 \mu \mathrm{g} \mathrm{ml}^{-1}$ hemin (Fluka, Buchs, Switzerland) and $0.5 \mu \mathrm{g} \mathrm{ml}^{-1}$ menadione (VWR International, Dietikon, Switzerland) and simulated body fluid [23] (1:1) supplemented with $0.2 \%$ glucose. Each biofilm-coated substratum was anaerobically incubated in a single well at $37^{\circ} \mathrm{C}$ for $18 \mathrm{~h}$, for an overall biofilm growth time of $90 \mathrm{~h}$.

\section{Toothbrush exposition}

Four toothbrushes with side-to-side action were selected according to the technical parameter of the number of head oscillations per minute. The oscillation frequencies were taken from the manufacturer's data. The selected toothbrushes were purchased in a store by one of the authors (JCS) and were labeled toothbrush A (Trisa ${ }^{\circledR}$ Sonic Impulse, Trisa Electronics AG, Triengen, Switzerland; 20,000 oscillations per minute), toothbrush B (Oral-B ${ }^{\circledR}$ Pulsonic Slim Type 3746, Braun GmbH, Kronberg, Germany; 27,000 oscillations per minute), toothbrush $\mathrm{C}$ (Philips ${ }^{\circledR}$ Sonicare FlexCare HX6902/02, Philips GmbH, Hamburg, Germany; 31,000 oscillations per minute), and toothbrush D (Waterpik ${ }^{\circledR}$ Sensonic $^{\circledR}$ Professional SR-1000E, Water Pik Inc., Fort Collins, CO, USA; 30,000 oscillations per minute). Each toothbrush was installed in an individually manufactured and adjustable toothbrush apparatus.

After biofilm formation, each disk was gently dipped in physiological saline to remove any nonadherent bacteria and was then placed in the exposure container of the toothbrush apparatus filled with physiological saline. The toothbrush was aligned toward the center of the disk in a stationary horizontal position with the following combinations of brushing time and distance between the end of the longest central bristles and the disk surface: $2 \mathrm{~s} / 0 \mathrm{~mm}, 2 \mathrm{~s} / 2 \mathrm{~mm}$, $2 \mathrm{~s} / 4 \mathrm{~mm}, 4 \mathrm{~s} / 2 \mathrm{~mm}$, and $6 \mathrm{~s} / 2 \mathrm{~mm}$ [8]. In a preliminary experiment, the distances between the bristles and the disk surface were determined by a ruler for each toothbrush. Each brushing position $(0,2$, and $4 \mathrm{~mm}$ distance) yielded a benchmark on the mounting stage of the toothbrush apparatus to adjust the toothbrushes precisely and reproducibly during the subsequent experiments. Untreated disks served as controls. The toothbrushes were fully charged before use, and the highest mode of action for each product was employed. After the toothbrush treatment, the disks were dipped in physiological saline and subsequently prepared for 4',6-diamidino-2phenylindole dihydrochloride (DAPI; Sigma-Aldrich, Buchs, Switzerland) staining and analysis under a confocal laser scanning microscope (CLSM; Carl Zeiss AG, Oberkochen, Germany).

\section{Microscopical analysis}

The biofilms were fixed in $4 \%$ paraformaldehyde (SigmaAldrich, Buchs, Switzerland) for $30 \mathrm{~min}$ at $4{ }^{\circ} \mathrm{C}$ and were washed once with phosphate-buffered saline (PBS). Next, the biofilm-associated bacteria were permeabilized by exposure to lysozyme (Sigma-Aldrich, Buchs, Switzerland; $70,000 \mathrm{U} \mathrm{ml}^{-1}$ ) for $3 \mathrm{~min}$ at room temperature and were rinsed with physiological saline. The biofilm-coated disks were then covered with DAPI solution for $3 \mathrm{~min}$.

After DAPI staining, the biofilm-coated disks were washed once with PBS, embedded in an inverted position in $10 \mu$ of Mowiol mounting medium, and stored at room temperature in the dark for at least $8 \mathrm{~h}$. The biofilms were examined under a Zeiss LSM700 inverted confocal microscope working through a vertical view. Images of $1,024 \times 1,024$ pixels in size 
were acquired using Zeiss ZEN 2010 software with the fluorescence signal assigned to a blue color. Confocal images were acquired using a $63 \times$ (numeric aperture 1.4) oil immersion plan apochromatic objective lens and a $405-\mathrm{nm}$ laser.

Three randomly selected microscopic fields near the center of the disk, each $0.021 \mathrm{~mm}^{2}$ in diameter (corresponding to $0.32 \%$ of the total surface area), were scanned. Vertical optical sectioning at every position with a slice thickness of $0.29 \mu \mathrm{m}$ was used to generate Z-direction series. The biofilm volumes were determined using volumetric analyses with Imaris version 7.5.2 software (Bitplane AG, Zurich, Switzerland). Three confocal datasets for each disk were analyzed, and the means and standard deviations of the biofilm volumes were calculated.

The mean volumes of the biofilms on the exposed substrates were compared to those of the unexposed control from the same experiment. The percent reduction or expansion of each biofilm was recorded. A biofilm volume of at least $15,000 \mu^{3}$ on the control disk was required for an experiment to be included in the analysis.

\section{Statistical analysis}

A total of 16 independent flow chamber experiments were used to generate 96 biofilm-coated titanium disks. Each of the 16 experiments included a control disk. A total of 80 biofilm-coated disks were randomly distributed to each experimental group, which was defined by the brushing time and brushing distance. Randomization was performed using a computer-generated list (Microsoft Office Excel ${ }^{\circledR}$ 2011, Microsoft Corp., Redmond, WA, USA).

The results of a Shapiro-Wilk test indicated that most of the data were not normally distributed. Therefore, a onesample Wilcoxon signed-rank test was applied. The null hypothesis was that the median percentage of biofilm reduction by noncontact brushing was zero.

All the calculations were performed using SPSS ${ }^{\circledR}$ software (SPSS ${ }^{\circledR}$ Statistics 20.0.0; SPSS Inc., Chicago, IL, USA). The differences in the percent biofilm reduction achieved by the different toothbrushes were evaluated using the Mann-Whitney $U$ test. A result was considered to be statistically significant if $p<0.05$.

\section{Results}

Impact of brushing time

The differences in biofilm reduction after various brushing times are shown in Fig. 1. A significant difference in biofilm reduction between toothbrushes $\mathrm{A}$ and $\mathrm{D}$ was observed after a brushing time of $2 \mathrm{~s}(p=0.029)$. There were no other significant differences in biofilm reduction after any other brushing times with the toothbrushes $(p>0.05)$.
Impact of brushing distance

The differences in biofilm reduction at various brushing distances are shown in Fig. 2. There was a significant difference between contact and noncontact brushing with $4 \mathrm{~mm}$ distance for toothbrush $\mathrm{C}(p=0.029)$. Moreover, a significant difference in biofilm reduction between toothbrushes $\mathrm{A}$ and D was observed at a brushing distance of $2 \mathrm{~mm}(p=0.029)$. There were no other significant differences between any other variables of the toothbrushes $(p>0.05)$.

\section{Overall efficacy}

The efficacy of the tested toothbrushes for noncontact biofilm removal is shown in Fig. 3. Significant differences in biofilm reduction were noted between toothbrushes $\mathrm{A}$ and $\mathrm{C}$, toothbrushes $\mathrm{A}$ and $\mathrm{D}$, as well as toothbrushes $\mathrm{B}$ and $\mathrm{D}$. There were no other significant differences between any other toothbrushes. The biofilms were significantly reduced by toothbrush $\mathrm{C}(p=0.001, n=16)$ and toothbrush $\mathrm{D}$ $(p=0.001, n=16)$. The reduction of biofilms was not significantly predictable for toothbrush A $(p=0.352, n=16)$ and toothbrush B ( $p=0.959, n=16)$.

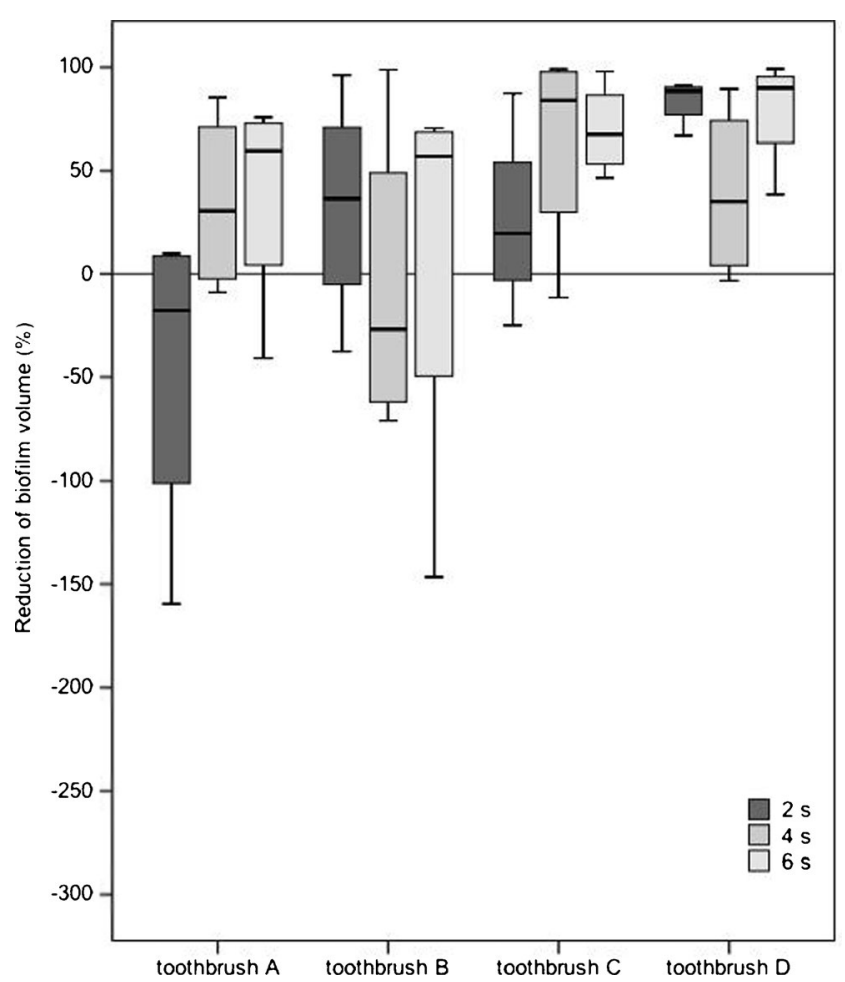

Fig. 1 Reduction in the biofilm volume (percent) after three different brushing times (at a brushing distance of $2 \mathrm{~mm}$ ). The negative values represent expansions of the biofilm. The boxplot indicates the medians, interquartile ranges (IQRs), and full range of values from four independent experiments $(n=4)$ 


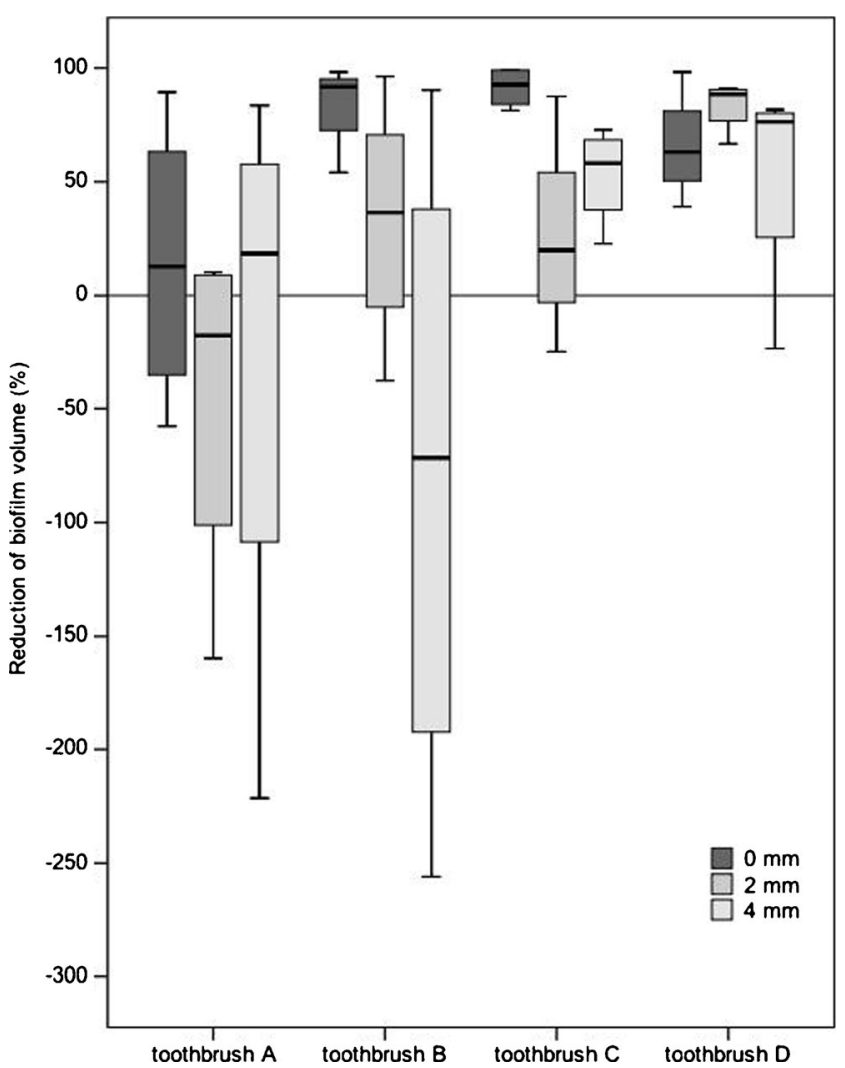

Fig. 2 Reduction in the biofilm volume (percent) at three different brushing distances (after a brushing time of $2 \mathrm{~s}$ ). The negative values represent expansions of the biofilm. The boxplot indicates the medians, IQRs, and full range of values from four independent experiments $(n=4)$

Microscopic images

Representative CLSM images of biofilms after a brushing time of $4 \mathrm{~s}$ are shown in Fig. 4. The effective reductions in biofilm volumes (compared to an unexposed control) are depicted for toothbrushes $\mathrm{C}$ and $\mathrm{D}$. In contrast, biofilm expansions are illustrated in the microscopic images from the experiments with toothbrushes $\mathrm{A}$ and $\mathrm{B}$.

\section{Discussion}

The present study evaluated the efficacy of four side-to-side toothbrushes for noncontact brushing in vitro. The toothbrushes were selected according to the technical parameter of the number of head oscillations, which ranged from 20,000 to 31,000 oscillations per minute. Because an oral hygiene session may include various brushing distances and times for different tooth surfaces, an evaluation of the overall efficacy of the tested toothbrushes may be helpful. The median percentages of biofilm reduction ranged from $9 \%$ to $80 \%$. The toothbrushes differed significantly in their capability for noncontact biofilm removal $(p<0.05)$. Significant biofilm reductions were achieved by two of the tested toothbrushes.

The present in vitro model is characterized as follows:

Previous in vitro studies of noncontact biofilm removal have used one or two species for biofilm formation [8]. In this study, three different oral bacteria were selected and included in a multispecies biofilm, probably increasing the biological plausibility and at the same time, however, increasing the microbial variability. The adhesion characteristics of bacteria in mono-species biofilms may differ from those of bacteria derived from dental biofilms caused by bacterial interactions [13]. However, the enormous variety of oral microflora, which can consist of over 700 different bacterial species, makes mimicking the intraoral situation in a laboratory biofilm model unfeasible [24, 25].

SLA titanium disks were used as a standardized substratum. The adhesion-promoting properties of SLA titanium disks may promote the initial bacterial colonization, which is thought to represent a critical phase of biofilm formation [26-28]. Thereafter, biofilm growth and maturation may occur independently of the underlying surface properties $[12,27]$.

A dynamic flow chamber system was employed for the initial biofilm growth. The dynamic conditions in the flow chamber system are intended to mimic the flow rate and shear forces of the saliva flow in the oral environment $[18,19]$. In contrast, static systems may enhance biofilm growth, leading to a more compact, multilayered biofilm $[14,29]$. The strengths of biofilms grown in static and dynamic systems may differ from each other [30, 31]. However, this investigation aimed to model a combination of the strengths of dynamic and static biofilm systems.

The brushing parameters were adapted by the clinical reality. Brushing times of up to $6 \mathrm{~s}$ were derived from calculations of the time available for cleaning a single tooth surface within an overall toothbrushing time of 2 to $3 \mathrm{~min}$ [8]. Distances of 2 and $4 \mathrm{~mm}$ from the longest central bristles to the biofilm-containing disk were used in the experiments.

Several groups have recently suggested that changes in detachment forces may mediate noncontact biofilm removal [8]. Adams et al. [32] observed the movements of fluid and air bubbles. Side-to-side and multidimensional toothbrushes generated similar shear forces despite having different biofilm removal efficacies, which indicates that hydrodynamic forces may not be the only important influence of biofilm removal. Because the mechanisms of noncontact biofilm removal have not been characterized in detail, however, it remains unclear which effects are responsible for this process. 
Fig. 3 Overall reduction in the biofilm volume (percent) after noncontact brushing (brushing times of 2, 4, and $6 \mathrm{~s}$; brushing distances of 2 and $4 \mathrm{~mm}$ ). The negative values represent expansions of the biofilm. The boxplot indicates the medians, IQRs, and full range of values from 16 independent experiments $(n=16)$. The data points denote outliers with IQRs greater than twice the median value. The statistically calculated differences (Mann-Whitney $U$ test) among the four analyzed toothbrushes are shown in the table

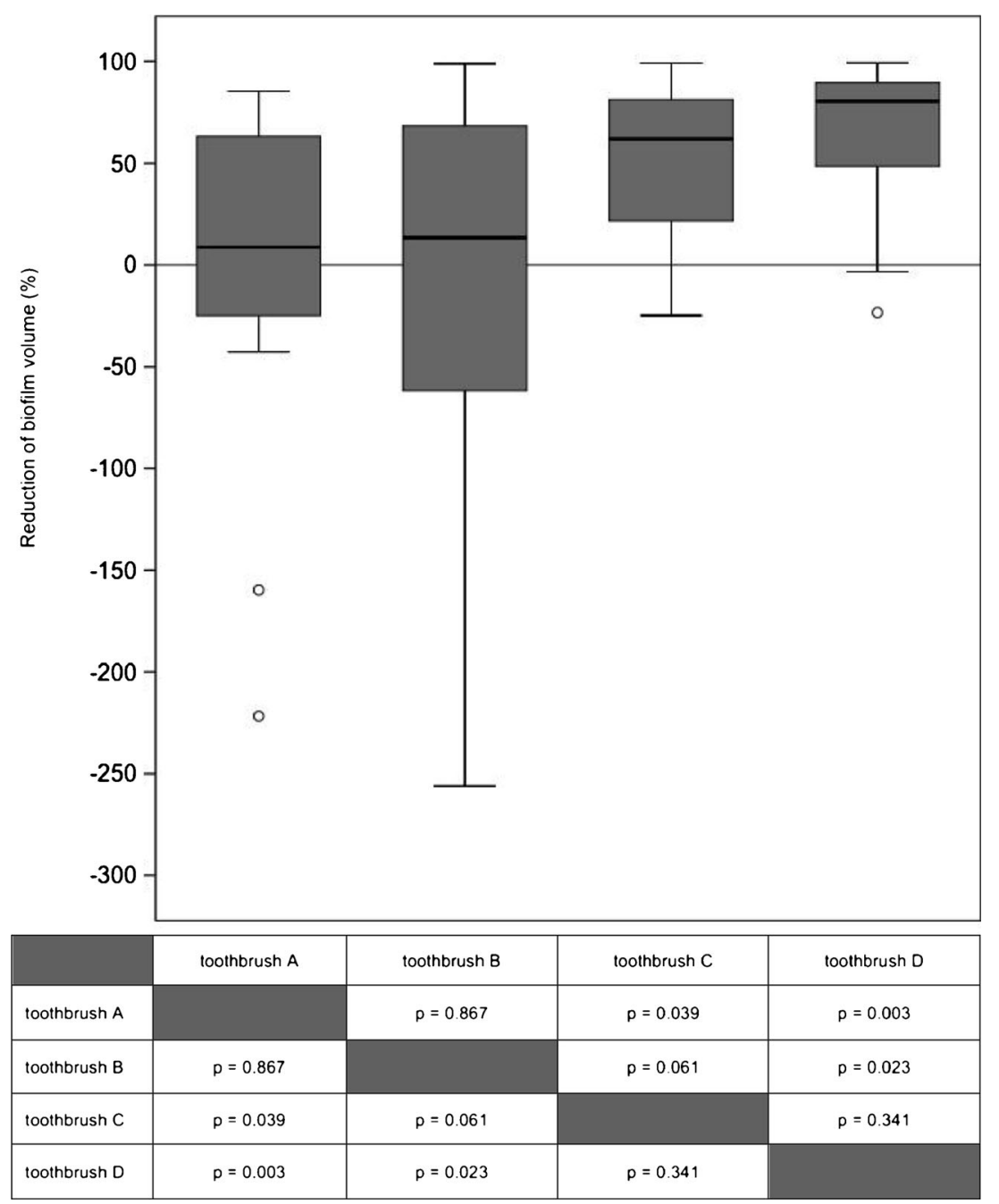

The interplay among (1) hydrodynamic effects in terms of shear forces, (2) thermodynamic surface tension forces caused by passing air bubbles, and (3) acoustic energy transfer in terms of sound pressure waves may be associated with noncontact biofilm removal [8].

Previous studies have failed to find a significant influence of brushing time on noncontact biofilm removal when the brushing distance is $\leq 2 \mathrm{~mm}$ [8]. The brushing times in these reports ranged from 5 to $30 \mathrm{~s}$, and the majority of biofilmassociated bacteria were removed within $5 \mathrm{~s}$. The present data demonstrated a trend toward greater biofilm reduction after $6 \mathrm{~s}$ compared to $2 \mathrm{~s}$. However, the impact of brushing time on noncontact biofilm removal was not significant when there was a brushing time per surface of 2 to $6 \mathrm{~s}$.

Increasing brushing distances have impeded noncontact biofilm removal by powered toothbrushes in recent studies [8]. No significant change in biofilm removal after an increase of the brushing distance from 2 to $4 \mathrm{~mm}$ was observed in the present study. This finding might be due to the shorter but more clinically relevant brushing time and the shorter distance of $4 \mathrm{~mm}$ employed in the current protocol compared to $6 \mathrm{~mm}$ in previous studies.

Under several conditions, two of the tested toothbrushes caused a volumetric expansion of the biofilm. A trend toward a greater degree of biofilm expansion was observed at a 4$\mathrm{mm}$ brushing distance compared to a 2-mm distance. These data are consistent with those of Busscher et al. [10], who reported that biofilm expansion occurred at distances of 4 and $6 \mathrm{~mm}$ using side-to-side and multidimensional brushes. Busscher et al. [10] have suggested that biofilm expansion occurs via a viscoelastic mechanism. The energy transfer from the toothbrush to the biofilm may lead to a plastic deformation manifested as an expansion of the biofilm [10]. The authors attributed the enhanced variability in their results to plastic deformation, which may be difficult to control. In the present study, biofilm expansion was also correlated with a greater variety in the results of repeated measurements, which was observed for toothbrushes A and $\mathrm{B}$ (the two toothbrushes with lower frequencies). In addition, an interesting phenomenon was observed in the CLSM 
Fig. 4 Representative CLSM images after a brushing time of $4 \mathrm{~s}$ at a brushing distance of $2 \mathrm{~mm}$ : overlaid images $(1-5)$ and cross-sections $(6-10)$ of unexposed biofilms (control) and biofilms treated with toothbrushes A, B, C, and D Bar $=10 \mu \mathrm{m}$
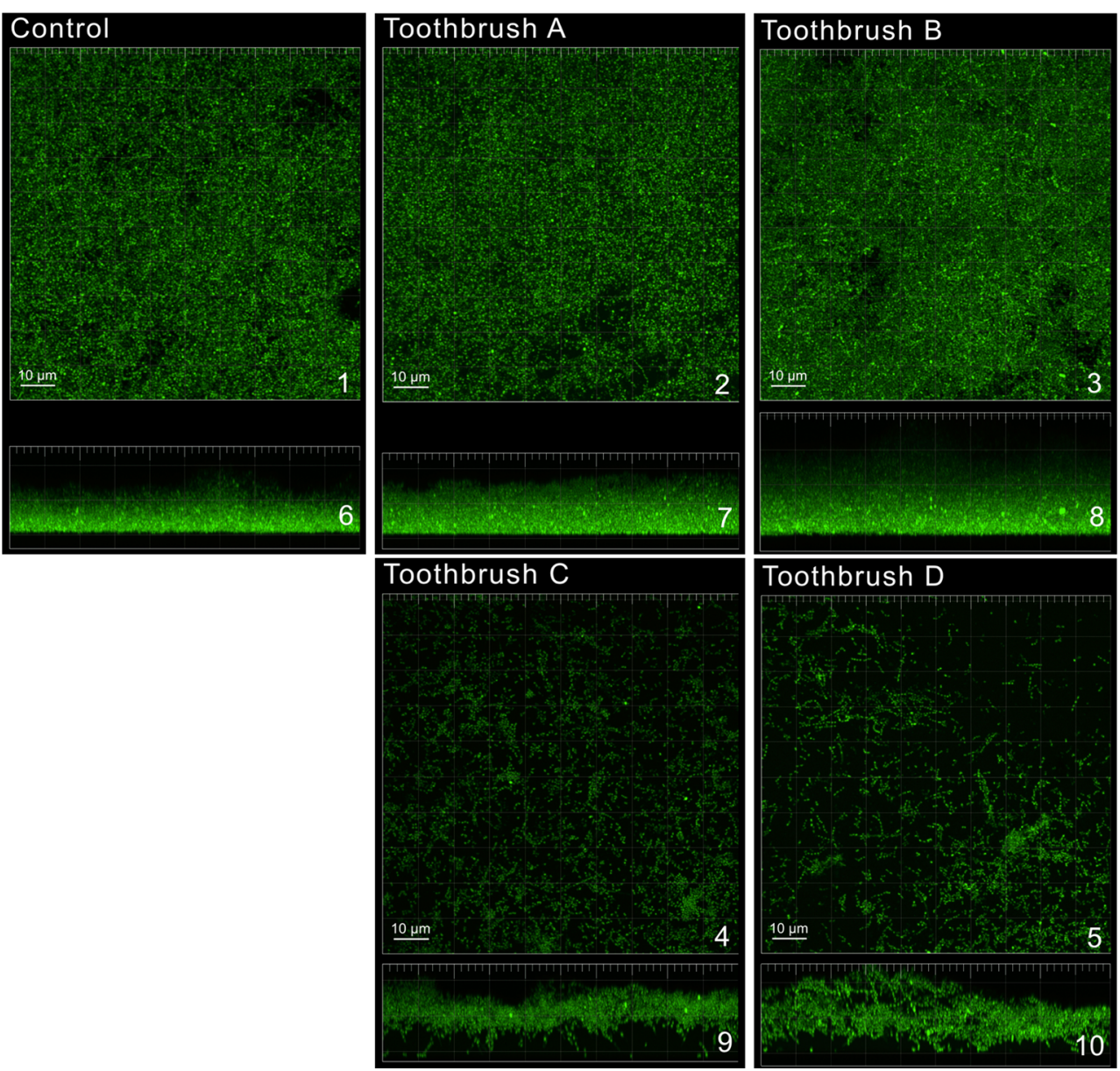

images for toothbrush $\mathrm{C}$ and $\mathrm{D}$, indicating an altered biofilm structure (Fig. 4). The relevance of this microscopically observed biofilm morphology is unknown. However, this finding requires further research.

Interestingly, a pronounced biofilm expansion was caused in the present study by the powered toothbrushes that operated at lower frequencies $(20,000$ and 27,000 head oscillations per minute for toothbrushes $\mathrm{A}$ and $\mathrm{B}$, respectively). Toothbrushes $\mathrm{C}$ and $\mathrm{D}$ had higher frequencies of 31,000 and 30,000 oscillations per minute, respectively. The biofilms were significantly reduced by toothbrush $\mathrm{C}$ and toothbrush D. Significant differences in the overall efficacy of biofilm reduction were noted between toothbrushes $\mathrm{A}$ and $\mathrm{C}$, toothbrushes $\mathrm{A}$ and $\mathrm{D}$, and between toothbrushes $\mathrm{B}$ and $\mathrm{D}$ indicating that oscillation frequency may be a factor in biofilm removal. However, the impacts of oscillation and of other technical parameters on biofilm removal were not investigated and remain a challenging aim for further research. An appropriate design is currently under preparation in our laboratory. The frequency and amplitude of the bristle vibrations, as well as the bristle design (for example, the number, configuration, length, and material of the bristles), are examples of factors that may influence noncontact biofilm removal. Until the impacts of these technical parameters are better understood, the interpretation of our results should be limited to the toothbrushes tested.

Previous studies have frequently reported noncontact biofilm removal levels of more than $50 \%$ by side-to-side toothbrushes [8]. This finding is in accordance with the present results of median biofilm reductions of $62 \%$ and $80 \%$ by toothbrushes $\mathrm{C}$ and $\mathrm{D}$, respectively. In contrast, toothbrushes $\mathrm{A}$ and $\mathrm{B}$ achieved biofilm reductions of greater than $50 \%$ in a minority of experiments, leading to median overall efficacies of $9 \%$ and $13 \%$, respectively.

The surface roughness of the SLA titanium disks differs from the physical properties of human tooth surfaces. However, a translation of the noncontact biofilm removal results from industrially manufactured surfaces to enamel or dentine surfaces may be possible due to several microbial similarities between periodontal and peri-implant lesions in humans [33, 34]. The standardized rough titanium surface was selected to promote the initial bacterial colonization [26, 27]. Industrially manufactured surfaces may help ensure the repeatability and reproducibility of biofilm formation, in contrast to nonstandardized tooth surfaces, which exhibit variable surface characteristics. In addition, collecting a reliable number of 
human teeth for research remains a challenge due to availability and ethical considerations. This study of noncontact biofilm removal may also be interpreted as a first study on the efficacy of side-to-side toothbrushes on exposed rough titanium surfaces. A rough titanium surface may be exposed after peri-implantitis or resective peri-implantitis treatment [35]. Two of the toothbrushes examined in this study were able to reduce a three-species biofilm on a rough titanium surface by noncontact brushing.

The present data were obtained in an in vitro environment. From a clinical perspective, the prevention and treatment of periodontal and peri-implant diseases, as well as the establishment of long-term oral health, require the correct daily performance of dental plaque removal by the patient [7]. Noncompliance with oral hygiene practices, however, is a major problem in self-performed oral hygiene, particularly in patients with lower socioeconomic status [36-39]. Inadequate compliance is correlated with the deterioration of the periodontal tissues, leading to periodontal or peri-implant diseases [40-43]. Powered toothbrushes with various modes of action have been developed to improve and simplify oral hygiene [44]. Currently, several models are commercially available; they vary in terms of technical parameters and sale prices.

It would therefore be desirable to examine the efficacy of toothbrushes for noncontact biofilm removal in clinical studies after demonstrating their efficacy in laboratory studies. A clinical setting is challenging; it may require appropriate follow-up visits and the standardization of indices and clinically relevant thresholds for differences in plaque and gingival health outcomes [45].

\section{Conclusions}

In conclusion, this study produced evidence that two of the tested side-to-side toothbrushes, i.e., $\mathrm{C}$ and $\mathrm{D}$, were able to reduce an in vitro biofilm by noncontact brushing. The efficacy of the tested toothbrushes for noncontact biofilm removal differed significantly. The extrapolation of these in vitro findings to powered toothbrushes that were not examined here is not recommended.

\begin{abstract}
Acknowledgments We thank Elisabeth Filipuzzi-Jenny (Clinic of Preventive Dentistry and Oral Microbiology, University of Basel) for laboratory assistance; Dr. Oliver Biehlmaier and Dr. Alexa Ferrand (Image Core Facility, Biozentrum, University of Basel) for assistance with microscopic analyses; and Ing. EurEta Sascha Martin (Department of Physics, University of Basel) for the construction of the toothbrush apparatus.
\end{abstract}

Conflicts of interest The authors declare that they have no conflicts of interest. This project was supported in part by an unrestricted grant by the Swiss Society of Dentistry (SSO), project number 264-12. The titanium disks were provided by Straumann AG (Basel, Switzerland).

\section{References}

1. Socransky SS, Haffajee AD (2000) Dental biofilms: difficult therapeutic targets. Periodontol 28:12-55

2. Del Pozo JL, Patel R (2007) The challenge of treating biofilmassociated bacterial infections. Clin Pharmacol Ther 82:204-209

3. Sanz M, van Winkelhoff AJ (2011) Periodontal infections: understanding the complexity - consensus of the Seventh European Workshop on Periodontology. J Clin Periodontol 38(Suppl 11):3-6

4. Kolenbrander PE, Palmer RJ Jr, Periasamy S, Jakubovics NS (2010) Oral multispecies biofilm development and the key role of cell-cell distance. Nat Rev Microbiol 8:471-480

5. Walter C, Weiger R (2006) Antibiotics as the only therapy of untreated chronic periodontitis: a critical commentary. J Clin Periodontol 33:938-939, author reply 940-931

6. Herrera D, Alonso B, Leon R, Roldan S, Sanz M (2008) Antimicrobial therapy in periodontitis: the use of systemic antimicrobials against the subgingival biofilm. J Clin Periodontol 35:45-66

7. Axelsson P, Nystrom B, Lindhe J (2004) The long-term effect of a plaque control program on tooth mortality, caries and periodontal disease in adults. Results after 30 years of maintenance. J Clin Periodontol 31:749-757

8. Schmidt JC, Zaugg C, Weiger R, Walter C (2013) Brushing without brushing? - a review of the efficacy of powered toothbrushes in noncontact biofilm removal. Clin Oral Investig 17:687-709

9. Sharma PK, Gibcus MJ, van der Mei HC, Busscher HJ (2005) Influence of fluid shear and microbubbles on bacterial detachment from a surface. Appl Environ Microbiol 71:3668-3673

10. Busscher HJ, Jager D, Finger G, Schaefer N, van der Mei HC (2010) Energy transfer, volumetric expansion, and removal of oral biofilms by non-contact brushing. Eur J Oral Sci 118:177-182

11. Hannig C, Hannig M (2009) The oral cavity-a key system to understand substratum-dependent bioadhesion on solid surfaces in man. Clin Oral Investig 13:123-139

12. Teles FR, Teles RP, Sachdeo A, Uzel NG, Song XQ, Torresyap G, Singh M, Papas A, Haffajee AD, Socransky SS (2012) Comparison of microbial changes in early redeveloping biofilms on natural teeth and dentures. J Periodontol 83:1139-1148

13. Palmer RJ Jr, Gordon SM, Cisar JO, Kolenbrander PE (2003) Coaggregation-mediated interactions of streptococci and actinomyces detected in initial human dental plaque. J Bacteriol 185:3400-3409

14. Paramonova E, Kalmykowa OJ, van der Mei HC, Busscher HJ, Sharma PK (2009) Impact of hydrodynamics on oral biofilm strength. J Dent Res 88:922-926

15. Lea SC, Khan A, Patanwala HS, Landini G, Walmsley AD (2007) The effects of load and toothpaste on powered toothbrush vibrations. J Dent 35:350-354

16. Saxer UP, Imfeld T, van Waes H (2010) Medienmitteilung "Hydrodynamik-Schallzahnbürsten". SSO Taskforce, Bern

17. Astasov-Frauenhoffer M, Braissant O, Hauser-Gerspach I, Daniels AU, Weiger R, Waltimo T (2012) Isothermal microcalorimetry provides new insights into biofilm variability and dynamics. FEMS Microbiol Lett 337:31-37

18. Hauser-Gerspach I, Kulik EM, Weiger R, Decker EM, Von Ohle C, Meyer J (2007) Adhesion of Streptococcus sanguinis to dental implant and restorative materials in vitro. Dent Mater J 26:361-366

19. Weiger R, Decker EM, Krastl G, Brecx M (1999) Deposition and retention of vital and dead Streptococcus sanguinis cells on glass surfaces in a flow-chamber system. Arch Oral Biol 44:621-628

20. Decker EM, Weiger R, Wiech I, Heide PE, Brecx M (2003) Comparison of antiadhesive and antibacterial effects of antiseptics on Streptococcus sanguinis. Eur J Oral Sci 111:144-148

21. Decker EM, Weiger R, von Ohle C, Wiech I, Brecx M (2003) Susceptibility of planktonic versus attached Streptococcus sanguinis cells to chlorhexidine. Clin Oral Investig 7:98-102 
22. Decker EM, Maier G, Axmann D, Brecx M, von Ohle C (2008) Effect of xylitol/chlorhexidine versus xylitol or chlorhexidine as single rinses on initial biofilm formation of cariogenic streptococci. Quintessence Int 39:17-22

23. Cho SB, Nakanishi K, Soga N, Ohtsuki C, Nakamura T, Kitsugi T, Yamamuro T (1995) Defence of apatite formation on silica gel on its structure: effect of heat treatment. J Am Ceram Soc 78:1769

24. Kazor CE, Mitchell PM, Lee AM, Stokes LN, Loesche WJ, Dewhirst FE, Paster BJ (2003) Diversity of bacterial populations on the tongue dorsa of patients with halitosis and healthy patients. $\mathrm{J}$ Clin Microbiol 41:558-563

25. Kinane DF, Hajishengallis G (2009) Polymicrobial infections, biofilms, and beyond. J Clin Periodontol 36:404-405

26. Rimondini L, Fare S, Brambilla E, Felloni A, Consonni C, Brossa F, Carrassi A (1997) The effect of surface roughness on early in vivo plaque colonization on titanium. J Periodontol 68:556-562

27. Dezelic T, Guggenheim B, Schmidlin PR (2009) Multi-species biofilm formation on dental materials and an adhesive patch. Oral Health Prev Dent 7:47-53

28. Bürgers R, Gerlach T, Hahnel S, Schwarz F, Handel G, Gosau M (2010) In vivo and in vitro biofilm formation on two different titanium implant surfaces. Clin Oral Implants Res 21:156-164

29. Stepanovic S, Vukovic D, Jezek P, Pavlovic M, Svabic-Vlahovic M (2001) Influence of dynamic conditions on biofilm formation by staphylococci. Eur J Clin Microbiol Infect Dis 20:502-504

30. Stoodley P, Cargo R, Rupp CJ, Wilson S, Klapper I (2002) Biofilm material properties as related to shear-induced deformation and detachment phenomena. J Ind Microbiol Biotechnol 29:361-367

31. Verkaik MJ, Busscher HJ, Rustema-Abbing M, Slomp AM, Abbas F, van der Mei HC (2010) Oral biofilm models for mechanical plaque removal. Clin Oral Investig 14:403-409

32. Adams H, Winston MT, Heersink J, Buckingham-Meyer KA, Costerton JW, Stoodley P (2002) Development of a laboratory model to assess the removal of biofilm from interproximal spaces by powered tooth brushing. Am J Dent 15:12B-17B

33. Leonhardt A, Adolfsson B, Lekholm U, Wikstrom M, Dahlen G (1993) A longitudinal microbiological study on osseointegrated titanium implants in partially edentulous patients. Clin Oral Implants Res 4:113-120
34. Heitz-Mayfield LJ, Lang NP (2010) Comparative biology of chronic and aggressive periodontitis vs. peri-implantitis. Periodontol 2000 53:167-181

35. Romeo E, Ghisolfi M, Murgolo N, Chiapasco M, Lops D, Vogel G (2005) Therapy of peri-implantitis with resective surgery. A 3-year clinical trial on rough screw-shaped oral implants. Part I: clinical outcome. Clin Oral Implants Res 16:9-18

36. Genco RJ, Ho AW, Grossi SG, Dunford RG, Tedesco LA (1999) Relationship of stress, distress and inadequate coping behaviors to periodontal disease. J Periodontol 70:711-723

37. Ak G, Sepet E, Pinar A, Aren G, Turan N (2005) Reasons for early loss of primary molars. Oral Health Prev Dent 3:113-117

38. Borrell LN, Burt BA, Warren RC, Neighbors HW (2006) The role of individual and neighborhood social factors on periodontitis: the third National Health and Nutrition Examination Survey. J Periodontol 77:444-453

39. Meisel P, Reifenberger J, Haase R, Nauck M, Bandt C, Kocher T (2008) Women are periodontally healthier than men, but why don't they have more teeth than men? Menopause 15:270-275

40. Quirynen M, De Soete M, van Steenberghe D (2002) Infectious risks for oral implants: a review of the literature. Clin Oral Implants Res 13:1-19

41. König J, Plagmann HC, Rühling A, Kocher T (2002) Tooth loss and pocket probing depths in compliant periodontally treated patients: a retrospective analysis. J Clin Periodontol 29:10921100

42. Eickholz P, Kaltschmitt J, Berbig J, Reitmeir P, Pretzl B (2008) Tooth loss after active periodontal therapy. 1: patient-related factors for risk, prognosis, and quality of outcome. J Clin Periodontol $35: 165-174$

43. Heitz-Mayfield LJ (2008) Peri-implant diseases: diagnosis and risk indicators. J Clin Periodontol 35:292-304

44. Emling RC, Yankell SL (1997) The application of sonic technology to oral hygiene: the third generation of powered toothbrushes. $\mathrm{J}$ Clin Dent 8:1-3

45. Robinson PG, Damien Walmsley A, Heanue M, Deacon S, Deery C, Glenny AM, Worthington H, Shaw W (2006) Quality of trials in a systematic review of powered toothbrushes: suggestions for future clinical trials. J Periodontol 77:1944-1953 